\title{
iTRAQ Proteomics Profiling of Regulatory Proteins During
}

\section{Oligodendrocyte Differentiation}

Mohit Raja Jain ${ }^{l}$, Tong Liu ${ }^{l}$, Teresa L. Wood ${ }^{2}$ and Hong Li $^{1 *}$

${ }^{1}$ Center for Advanced Proteomics Research and Department of Biochemistry and Molecular

Biology, University of Medicine and Dentistry of New Jersey -New Jersey Medical School

Cancer Center, Newark, NJ 07103

${ }^{2}$ Department of Neurology and Neuroscience, University of Medicine and Dentistry of New Jersey -New Jersey Medical School Cancer Center, Newark, NJ 07103

Running Head: iTRAQ Proteomics Study of Differentiation

*Address correspondence to: Hong Li, Department of Biochemistry and Molecular Biology, University of Medicine and Dentistry of New Jersey-New Jersey Medical School Cancer Center, 205 South Orange Avenue, F1226, Newark, NJ 07103. Tel: 973-972-8396, Fax: 973-972-5594, E-Mail: liho2@umdnj.edu 


\section{i. Summary}

Recent evolution in proteomics approaches from 2-dimensional gel electrophoresis to peptidebased "shotgun proteomics" methods has greatly enhanced the abilities of scientists to uncover expression changes among "low abundant" proteins. Shotgun proteomics methods typically employ stable isotope labeling techniques to distinguish peptides from the various sources that are compared. Recently, a new shotgun quantitative proteomics technology called isobaric tags for relative and absolute quantification (iTRAQ) has been developed for protein expression analysis. The major strength of the iTRAQ technology is its ability to compare the proteomic changes among multiple samples in a single experiment. Here we present a protocol on using the eight-plex iTRAQ approach for the discovery of molecular targets in oligodendrocyte progenitor cells during rapamycin-induced inhibition of differentiation. We would provide the technical details on peptide labeling, chromatography, mass spectrometry, database search and bioinformatics procedures for the identification of differentially expressed proteins. 


\section{ii. Key Words}

Mass spectrometery, iTRAQ, neuroproteomics, expression proteomics, mTOR, oligodendrocyte progenitor cell, rapamycin 


\section{Introduction}

Systems biological approaches are increasingly used as unbiased discovery tools to gain insights into the functional molecular alterations during neurological diseases (1). Currently microarray analysis has been a method of choice for "system-wide" gene expression analysis; however the application of microarray approaches has limitations in select biological scenerios, e.g., in cerebrospinal fluids which are mostly devoid of mRNAs. Furthermore, mRNA changes do not always correlate directly to changes at either protein levels or activities as the result of varying regulatory steps during translation, post-translational modifications, sub-cellular localization and degradation (2). An evolving approach towards comprehensive understanding of the molecular mechanisms underlying neurological diseases is shotgun neuroproteomics, i.e., the large-scale quantification of peptides and proteins within the contexts of stem cell differentiation, neurodegeneration, neuroregeneration, etc $(3,4)$. Accurate quantification of peptides relies on the availability of high resolution mass spectrometers that are increasingly robust and are equipped with sophisticated bioinformatic tools for routine high-throughput analysis. As an example of earlier shotgun methods, isotope-coded affinity tags (ICAT) reagents are used to label proteins (5). They are thiol-reactive chemical "tags" that are designed to contain either a light $\left[{ }^{12} \mathrm{C}\right]$ or heavy $\left[{ }^{13} \mathrm{C}\right]$ ICAT reagent with a mass difference of 9 Da. Relative protein abundance is determined from the relative MS ion abundance of the corresponding ICAT-labeled peptides. Since the ICAT method selectively quantifies only cysteine-containing peptides, it is able to quantify both protein expression and oxidative modification changes, but unable to quantify proteins that do not contain cysteines $(6,7)$. Alternatively, in stable isotope labeling by

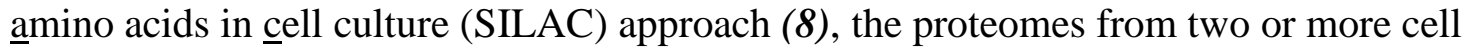


populations are compared which are metabolically labeled during protein synthesis with either light or heavy stable isotope- incorporated amino acids during cell culture. Quantification of proteins is achieved by comparing the relative abundance of peptides with identical sequences, yet distinguishable in MS by their relative mass due to "heavy" amino acid incorporation.

Although SILAC has been proven effective for large scale quantification of proteins and their phosphorylation changes, it cannot be readily used for studying terminally differentiated cells including neurons since they are not rapidly dividing to incorporate SILAC-specific amino acids into proteins. In addition, this method is not suitable for routine analysis of tissues. By comparison, the iTRAQ approach has been successfully used for multiplexed protein expression analysis from both animal tissues and neuronal cells (9-15). This method utilizes the covalent labeling of peptides with isobaric mass tags for simultaneous identification and quantification of peptides derived from up to eight categories using tandem mass spectrometry (MS/MS) methods. iTRAQ reagents are identical in mass, therefore isobaric, and are consisted of a variety of isotope-incorporated reporter groups, corresponding stable isotope mass balance groups, and a peptide reactive group. These reagents are covalently linked with peptides via primary amines at lysine side chains and N-termini. The iTRAQ-labeled peptides from up to eight samples can be combined, fractionated using two-dimensional liquid chormatography (LC) and identified and quantified using MS/MS. iTRAQ-labeled peptides labeled with any one of the eight iTRAQ tags are eluted at the same retention time during the LC steps and they display identical mass during MS analysis because they are isobaric. During MS/MS peptide fragmentation, the reporter ions that contain different combinations of stable isotopes $(\mathrm{m} / \mathrm{z} 113,114,115,116,117,118,119$ and 121) are released from the iTRAQ-tagged peptides. The peak areas of the reporter ions are used to determine the relative abundance of each peptide and of the corresponding proteins in each 
sample. A series of peptide $y$ - and b-ions fragments is also generated by MS/MS analysis for protein identification through protein database matching $(\mathbf{1 6}, \mathbf{1 7})$. Since iTRAQ reagents are efficient at labeling nearly all peptides, this method is effective at providing high protein identification sequence coverages, allowing sensitive quantification of low-abundant proteins such as signal transducers, transcription regulators and membrane receptors $(10-12,14)$.

There are currently two versions of iTRAQ reagents that are available from ABSciex (Foster City, CA) for the comparison of either four or eight sample categories simultaneously. We will present here an 8-plex iTRAQ-based shotgun neuroproteomics method that we have used to elucidate the target pathways downstream from mammalian target of rapamycin (mTOR) in oligodendrocyte progenitor cells (OPCs), following rapamycin induced inhibition of OPC differentiation. mTOR is a member of the phosphatidylinositol 3-kinase-like family of serinethreonine kinases that integrates signals from growth factor stimulation and nutrient sensing to modulate a number of biological processes including cell growth, proliferation, protein translation, differentiaton and autophagy (18). mTOR forms two intracellular signaling complexes known as mTOR Complex 1 (mTORC1) and mTOR Complex 2 (mTORC2), defined respectively by the association of $\mathrm{mTOR}$ with the adaptor proteins raptor or rictor that direct mTOR's kinase activity towards distinct downstream signaling effectors $(19,20)$. Inhibition of mTORC2 during OPC differentiation causes a reduction in the mRNA levels of several key myelin genes, whereas inhibiting mTORC1 results in decreased myelin protein levels most likely by interfering with the translation of these transcripts (21). By comparison, recent reports have shown that the rate of protein translation increases during OPC differentiation in an mTORdependent fashion (22). However, the targets of the mTOR pathway that regulate 
oligodendrocyte differentiation are unknown. To identify novel targets regulated by the mTOR pathway during oligodendrocyte differentiation, proteins from four independent control and rapamycin-treated OPCs are processed sequentially with disulfide reduction, alkylation and trypsin digestion (Fig. 1). The resulting peptides are individually labeled with the iTRAQ reagents. The four iTRAQ reagents $(113,114,115$ and 116) are utilized to label the peptides derived from the four control samples, and another four iTRAQ reagents (117, 118, 119 and 121) are used for labeling the peptides derived from rapamycin-treated samples. Equal amounts of the labeled peptides are combined and quantified using two-dimensional liquid chormatography coupled with tandem mass spectrometry.

\section{Materials}

\subsection{Protein Extraction}

1. Control and rapamycin treated OPCs

2. Phosphate buffer saline (PBS): $1.54 \mathrm{mM}$ Potassium phosphate monobasic $\left(\mathrm{KH}_{2} \mathrm{PO}_{4}\right)$, $155.17 \mathrm{mM}$ Sodium chloride $(\mathrm{NaCl}), 2.71 \mathrm{mM}$ Sodium phosphate dibasic $\left(\mathrm{Na}_{2} \mathrm{HPO}_{4^{-}}\right.$ $\left.7 \mathrm{H}_{2} \mathrm{O}\right)$

3. Lysis buffer: $500 \mathrm{mM}$ Triethylammonium bicarbonate (TEAB), $1.0 \%$ Igepal CA630 (NP-40), $1.0 \%$ Triton X-100, 0.1\% v/v each of protease inhibitor cocktail (Sigma, St. Louis, MO), phosphatase inhibitor cocktail 1 (Sigma, St. Louis, MO) and phosphatase inhibitor cocktail 2 (Sigma, St. Louis, MO), pH 8.5

4. Ultrasonic Homogenizer with 5/32" Micro-Tip (Omni International, Kennesaw, GA)

5. Ice bath

6. Micro centrifuge 5415 R (Eppendorf, Hauppauge, NY) 


\subsection{Protein Estimation}

1. BCA protein assay kit (Thermo Scientific Pierce, Rockford, IL)

2. Protein standard $(2.0 \mathrm{mg} / \mathrm{ml}$ bovine serum albumin $)$

3. SpectraMax 190 micro plate reader (Molecular Devices, Sunnyvale, CA)

\subsection{Eight-plex iTRAQ Labeling}

1. Reducing reagent: $50 \mathrm{mM}$ tris-(2-carboxyethyl) phosphine (TCEP) (AB SCIEX, Foster City, CA)

2. Cysteine alkylation reagent: $200 \mathrm{mM}$ methyl methanethiosulfonate (MMTS) (AB SCIEX, Foster City, CA)

3. HPLC grade isopropanol

4. HPLC grade water

5. Trypsin $(20 \mu \mathrm{g} / \mathrm{vial}$, Promega, Madison, WI $)$

6. iTRAQ reagents: 113, 114, 115, 116, 117, 118, 119 and 121 (Part Number 4390811, AB SCIEX, Foster City, CA)

7. Vacuum concentrator 5301 (Eppendorf, Hauppauge, NY)

\subsection{Strong Cation Exchange Liquid Chromatography (SCXLC)}

1. PerSeptive BioCAD SPRINT Perfusion chromatography system (PerSeptive Biosystems, Cambridge, MA)

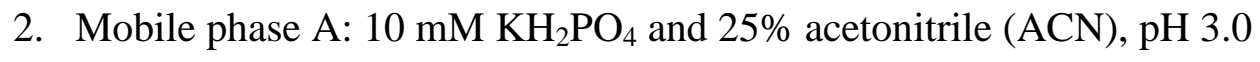

3. Mobile phase B: $500 \mathrm{mM} \mathrm{KCl}, 10 \mathrm{mM} \mathrm{KH}_{2} \mathrm{PO}_{4}$ and $25 \% \mathrm{ACN}$, pH 3.0

4. Mobile Phase C: $600 \mathrm{mM} \mathrm{KCl,} 10 \mathrm{mM} \mathrm{KH} 2 \mathrm{PO} 4$ and $25 \%$ ACN, pH 6.0

5. Column: Polysulfoethyl-A column $(4.6 \mathrm{~mm} \times 200 \mathrm{~mm}$, particle size $5.0 \mu \mathrm{m}, 300 \AA$ (Poly LC Inc., Columbia, MD) 
6. $\mathrm{pH}$ paper (Whatman Inc., Piscataway, NJ)

7. Two-ml fraction collection tube

\subsection{Peptide Desalting}

1. PepClean $\mathrm{C}_{18}$ spin columns (Thermo Scientific Pierce, Rockford, IL)

2. Activation solution: $50 \% \mathrm{ACN}$

3. Equilibration solution: $5 \% \mathrm{ACN}$ containing $0.5 \%$ trifluoroacetic acid (TFA)

4. Elution solution: $70 \% \mathrm{ACN}$ containing $0.1 \% \mathrm{TFA}$

\subsection{Reversed-Phase Liquid Chromatography (RPLC)}

1. Solvent A: $2 \%$ ACN containing $0.1 \%$ TFA

2. Solvent B: $85 \%$ ACN containing $0.1 \%$ TFA

3. Matrix-assisted laser desorption ionization (MALDI) matrix solution: $6 \mathrm{mg} / \mathrm{ml} \alpha$ cyano-4-hydroxycinnamic acid (Sigma, St Louis, MO) in 50\% ACN, 5 mM monobasic ammonium phosphate and internal calibrants (50 fmol/ml each of [Glu1]Fibrinopeptide B (GluFib), m/z 1570.677 (Sigma, St Louis, MO) and adrenocorticotropic hormone 18-39 (ACTH 18-39), m/z 2465.199, (Sigma, St Louis, $\mathrm{MO})$

4. LC-Packings Ultimate chromatography system equipped with a Probot MALDI spotting device (Dionex, Sunnyvale, CA, USA)

5. $\mathrm{C}_{18}$ PepMap trapping column (0.3 mm i.d. $\times 5 \mathrm{~mm}$ length, $5 \mu \mathrm{m}, 100 \AA ̊$, Dionex, Sunnyvale, CA, USA)

6. $\mathrm{C}_{18}$ PepMap capillary column $(0.1 \mathrm{~mm}$ i.d. $\times 150 \mathrm{~mm}$ length, $3 \mu \mathrm{m}, 100 \AA$ A, Dionex, Sunnyvale, CA, USA) 


\subsection{Mass Spectrometry}

1. 4800 Plus MALDI TOF/TOF Analyzer (AB SCIEX, Foster City, CA)

2. MALDI plates (AB SCIEX, Foster City, CA)

3. Mass standards kit (AB SCIEX, Foster City, CA)

4. 4000 Series Explorer (AB SCIEX, Foster City, CA)

\subsection{Data Analysis Software}

1. ProteinPilot (AB SCIEX, Foster City, CA, http://www.absciex.com)

2. Scaffold (Proteome Software Inc., Portland, OR, http://www.proteomesoftware.com)

3. TS2Mascot (Matrix Science Inc., Boston, MA, http://www.matrixscience.com)

4. Mascot (Matrix Science Inc., Boston, MA, http://www.matrixscience.com)

5. Excel (Microsoft corporation, Redmond, WA, http://office.microsoft.com)

\section{Methods}

\subsection{Protein Extraction:}

1. For each sample category, wash $5 \times 10^{6}$ cells with ice cold PBS twice to remove any residual media (see Note 1).

2. Carefully harvest the cells in $1 \mathrm{ml}$ of PBS with a corning cell scraper.

3. Pellet the cells in a micro centrifuge at $2,500 \times \mathrm{g}$ at $4{ }^{\circ} \mathrm{C}$ and remove the PBS completely (see Note 1).

4. Re-suspend each cell pellet in $250 \mu 1$ of cold iTRAQ lysis buffer by vortexing for 15 sec at high speed (see Note 2).

5. Lyse the cells by a 10 -sec sonication pulse followed by a 30 -sec incubation in the ice bath. Repeat three times. Incubate the cell lysate on the ice bath for $10 \mathrm{~min}$. 
6. Clarify the cell lysate by centrifugation at $16,100 \times \mathrm{g}$ for $30 \mathrm{~min}$ at $4{ }^{\circ} \mathrm{C}$ in a micro centrifuge.

7. Carefully transfer the supernatant to a fresh Eppendorf tube.

8. Estimate the protein concentrations using the BCA protein assay kit with bovine serum albumin protein standard diluted in the iTRAQ lysis buffer as the standards. Adjust the protein concentrations of all eight samples to same level by diluting the samples in the iTRAQ lysis buffer (see Note 3).

\subsection{Protein Digestion with Trypsin}

1. Transfer $75 \mu \mathrm{g}$ of proteins from each of the eight samples into a fresh tubes (see Note 4).

2. Add $2.0 \mu \mathrm{l}$ of the reducing reagent to each sample. Mix well by vortexing for $15 \mathrm{sec}$ and centrifuge briefly to bring down the solution (see Note 4).

3. Incubate the mixture at $60^{\circ} \mathrm{C}$ for $1 \mathrm{hr}$ with mixing (see Note 4).

4. Add $1 \mu \mathrm{l}$ of the cysteine alkylating solution. Mix well by vortexing for $15 \mathrm{sec}$ and centrifuge briefly to bring down the solution. Incubate the solution for $10 \mathrm{~min}$ at room temperature (see Note 4).

5. Reconstitute two vials $(20 \mu \mathrm{g} / \mathrm{vial})$ of sequencing grade trypsin with $80 \mu \mathrm{l}$ each in HPLC grade water by slowly pipetting the solution up and down a few times. Vortex for $30 \mathrm{sec}$ and centrifuge briefly to bring down the solution (see Note 5).

6. Add $20 \mu \mathrm{l}$ of the trypsin solution to each sample tube. Vortex for $1 \mathrm{~min}$ and centrifuge briefly to bring the solution down. Incubate the digestion reaction vials at $37^{\circ} \mathrm{C}$ for $16 \mathrm{hrs}$ in a water bath. Centrifuge briefly to bring all the solution down at the bottom (see Note 6). 


\subsection{Peptide Labeling with the 8-plex iTRAQ Reagents}

1. Bring the iTRAQ reagents out of the freezer to room temperature. Centrifuge briefly to bring the solution to the bottom of the vial (see Note 7).

2. Add $100 \mu \mathrm{L}$ of HPLC grade isopropanol to each vial of the iTRAQ reagent.

3. Vortex each vial at high speed for $30 \mathrm{sec}$ and then centrifuge briefly.

4. Transfer the entire contents of each freshly prepared iTRAQ reagent to their respective tryptic peptide sample tube. Vortex all the tubes at high speed for $30 \mathrm{sec}$ and then centrifuge briefly (see Note 8 ).

5. Test the $\mathrm{pH}$ of each sample by placing $0.5 \mu \mathrm{L}$ of the solution onto a $\mathrm{pH}$ paper. If necessary, add up to $5 \mu \mathrm{L}$ of $0.5 \mathrm{M}$ TEAB to adjust the $\mathrm{pH}$ of the final solution to between 7.5 and 8.5 (see Note 9).

6. Incubate the iTRAQ labeling reaction tubes at room temperature for $2 \mathrm{hrs.} \mathrm{Vortex} \mathrm{and}$ spin down briefly to bring solution to the bottom of the tubes (see Note 10).

7. Combine the contents of all eight iTRAQ labeled samples. Vortex to mix, then spin down the solution (see Note11).

8. Completely dry the combined sample in a speed vac at room temperature (see Note 12).

\subsection{Two-Dimension LC Separation of Peptides}

The combined iTRAQ-labeled peptides are fractionated first with strong cation exchange chromatography and then with reversed phase chromatography.

\subsubsection{Strong Cation Exchange Liquid Chromatograpy (SCXLC)}


Various reagents (e.g. TEAB, isopropanol, TCEP, detergents and excess iTRAQ reagents) used during protein extraction, digestion and labeling may interfere with either reversed-phase liquid chromatography steps or MS identification and quantification steps; therefore they must be removed completely beforehand. Peptide mixture is initially fractionated on a PerSeptive BioCAD SPRINT Perfusion chromatography system equipped with a PolySULFOETHYL A strong cation exchange column.

1. Reconstitute the iTRAQ labeled peptide mixture by adding $4 \mathrm{ml}$ of the SCXLC mobile phase A (see Note 13). Test the $\mathrm{pH}$ of the peptide solution using a $\mathrm{pH}$ paper. If necessary, adjust the $\mathrm{pH}$ of the solution to between 2.7-3.0 by addition of $1 \mathrm{M}$ phosphoric acid.

2. Centrifuge the sample at $20,000 \times \mathrm{g}$ for $15 \mathrm{~min}$ at $25^{\circ} \mathrm{C}$ to pellet the precipitates and particulates. Carefully transfer the clarified solution into a fresh tube.

3. Equilibrate the column for 15 column volume $(\sim 30 \mathrm{~mL})$ with the mobile phase $\mathrm{A}$.

4. Inject the iTRAQ-labeled peptides onto the SCXLC column through a $5 \mathrm{ml}$ sample loading loop (see Note 14).

5. After the injection, wash the column with 15 column volume $(\sim 30)$ with mobile phase A to remove the unbound iTRAQ reagents and detergents (see Note 15).

6. Elute the peptides with a 2 -segment linear gradient at a flow rate of $1 \mathrm{ml} / \mathrm{min}$ according to table below. Collect 2-min fractions during SCXLC in Eppendorf tubes (see Note 16).

\begin{tabular}{|l|l|l|l|}
\hline Time (min) & Mobile phase A (\%) & Mobile phase B (\%) & Mobile phase C (\%) \\
\hline 0 & 100 & 0 & 0 \\
\hline 45 & 50 & 50 & 0 \\
\hline
\end{tabular}




\begin{tabular}{|l|l|l|l|}
\hline 60 & 0 & 0 & 100 \\
\hline 75 & 0 & 0 & 100 \\
\hline
\end{tabular}

7. Dry each fraction completely in a speed vac.

\subsubsection{Concentration and Desalting the SCXLC Fractions with $\mathrm{C}_{18}$ Spin Column}

1. Re-suspend the peptides in each SCXLC fraction in $200 \mu 1$ of the equilibration solution (see Note 17). Sonicate in a water bath for $15 \mathrm{sec}$, vortex and then spin briefly.

2. Activate the $\mathrm{C}_{18}$ resin by adding $200 \mu$ of activation solution to the $\mathrm{C}_{18}$ spin columns. Centrifuge at $1,500 \times \mathrm{g}$ for $1 \mathrm{~min}$. Repeat this step 2 once more.

3. Equilibrate the $\mathrm{C}_{18}$ resin by adding $200 \mu \mathrm{l}$ of the equilibration solution. Centrifuge at $1,500 \times \mathrm{g}$ for $1 \mathrm{~min}$. Repeat this step 3 once more.

4. For each re-suspended SCXLC fraction, transfer the solution completely onto an equilibrated $\mathrm{C}_{18}$ spin column. Centrifuge at $1,500 \times \mathrm{g}$ for $1 \mathrm{~min}$. Collect the flow through and load the flow through again onto the spin column. Centrifuge at 1,500 $\times$ $\mathrm{g}$ for $1 \mathrm{~min}$. Repeat the step 4 once more.

5. Wash off the unbound salts by adding $200 \mu \mathrm{l}$ of the equilibration solution onto the column. Centrifuge at $1,500 \times \mathrm{g}$ for $1 \mathrm{~min}$. Repeat step 5 twice.

6. To elute the peptides, add $30 \mu \mathrm{l}$ of the elution solution onto the column. Collect the eluted peptides by centrifugation at 1,500 $\times \mathrm{g}$ for $1 \mathrm{~min}$ in a fresh Eppendorf tube. Repeat this step 6 twice and collect all the eluted peptides from each SCXLC fraction in same tube. 
7. Dry the desalted peptides in speed vac for further fractionation using reversed-phase liquid chromatography.

\subsubsection{Reversed-Phase Liquid Chromatography (RPLC)}

1. Reconstitute the peptides in each SCXLC fraction in $20 \mu \mathrm{l}$ of RPLC solvent A.

Vortex at high speed for $1 \mathrm{~min}$ and then spin briefly. Sonicate the sample in a water bath for $15 \mathrm{sec}$ and vortex. Centrifuge the tubes at $16,100 \times \mathrm{g}$ for $5 \mathrm{~min}$. Transfer each sample solution into the bottom of an auto-sampler vial and place all the vials in cooled auto sampler tray.

2. Equilibrate the RPLC column for at least 20 min with $2 \%$ solvent $B$ at $0.300 \mu 1 / \mathrm{min}$.

3. For each SCXLC fraction, load $5 \mu$ of the reconstituted peptides onto a $\mathrm{C}_{18}$ trapping column using a microliter pickup injection method at a flow rate of $20 \mu \mathrm{l} / \mathrm{min}$ (see

Note 18). Subsequently, the bound peptides are resolved in a high resolution $\mathrm{C}_{18}$ PepMap column at a flow rate of $0.3 \mu \mathrm{l} / \mathrm{min}$ with the following gradient.

\begin{tabular}{|l|l|l|}
\hline Time (min) & Solvent A (\%) & Solvent B (\%) \\
\hline 0 & 98 & 2 \\
\hline 6 & 98 & 2 \\
\hline 7 & 90 & 10 \\
\hline 51 & 77 & 23 \\
\hline 77 & 54 & 46 \\
\hline 89 & 5 & 95 \\
\hline 99 & 5 & 95 \\
\hline 100 & 98 & 2 \\
\hline
\end{tabular}




\section{1}

98

2

4. The eluted peptides are mixed with the MALDI matrix solution in a 1:1 ratio through a $30 \mathrm{~nL}$ mixing tee and directly spotted onto a MALDI plates in a $33 \times 10$ spot array format using Probot, which produces a spot every $12.5 \mathrm{sec}$ (see Note 19).

5. Repeat the RPLC steps for each of the SCXLC fractions.

\subsection{Mass Spectrometry:}

Peptides spotted on the MALDI plates are analyzed on a 4800 Plus MALDI TOF/TOF Analyzer using 4000 Series Explorer Software.

1. Tune and optimize the sensitivity and resolution of the mass spectrometer using the mass standard mixture kit. Check and optimize both metastable ion suppressor and the timed-ion-selector for specific precursor ion selection at the maximum resolution of 400 , corresponding to $\pm 2.5 \mathrm{Da}$ at $\mathrm{m} / \mathrm{z}$ of 1000 . Optimal performing instrument is very important for accurate iTRAQ quantification outcome (13).

2. Using the 6 peptide masses within the mass standard mixture kit, update all three MS calibration parameters (Detector Offset, TOF Offset, B-Factor) of the instrument using update default calibration function to ensure maximum mass accuracy. Update the MS/MS calibration parameters (Detector Offset, TOF Offset, B-Factor) using the MS/MS ion spectra of the GluFib ( $\mathrm{m} / \mathrm{z}$ 1570.677). (see Note 20)

3. For each quantitative project, create a new project on the 4000 Series Explorer Software. Then create a new spot set using a predefined spot set template. Load the sample MALDI plate into the mass spectrometer using the newly established spot set. 
4. Align the plate using the alignment MALDI spots specified in the corresponding spot set template. Confirm that the laser target crosshair in the video viewer is aligned with the laser spot.

5. Create an acquisition, a processing and a job-wide interpretation method for both MS and MS/MS analyses.

6. For the MS acquisition method, use positive MS reflector as the operating mode. Specify the mass range of interest as $\mathrm{m} / \mathrm{z}, 850-3,000$ and the focus mass as $1950 \mathrm{~m} / \mathrm{z}$. Set the laser intensity to 3,000 and the detector voltage multiplier at 0.90. Each MS spectrum is averaged over 1,000 laser shots. In the processing method, GluFib $(\mathrm{m} / \mathrm{z}$ 1570.677) and ACTH 18-39 ( $\mathrm{m} / \mathrm{z}$ 2465.199) masses are used as the internal calibrants. For the interpretation method, set following criteria for precursor selection for the subsequent MS/MS analysis; fifteen most abundant precursors per spot, minimum $\mathrm{S} / \mathrm{N}$ filter at 50, spot to spot precursor mass tolerance at $200 \mathrm{ppm}$ and from the rarest to the most abundant MS/MS ion as the data acquisition order.

7. For MS/MS acquisition method, use $2 \mathrm{KV}$ positive MS-MS method as the operating mode. Set the laser intensity to 4,000 and detector voltage multiplier at 0.90 . Specify the metastable suppression as "on", CID as "on" and the precursor mass window at relative 400 resolution (FWHM). Each MS/MS spectrum is accumulated over 2,000 laser shots. In the MS/MS processing method, each spectrum is smoothed using the Savitsky-Golay algorithm with points across peak set at 3 and polynomial order set at 4.

8. Set the medium CID gas recharge pressure to medium with a threshold of $5.0 \times 10^{7}$ torr. 


\subsection{Bioinformatics}

Peptide identification and quantification is determined by ProteinPilot software (v. 2.0.1) against the rat IPI database (v3.55, Release date Feb 12, 2009, 39,874 sequence entries) using the Paragon algorithm (23) in the search engine.

\subsubsection{Protein Identification}

1. The following default parameters are used for peptide identification by the ProteinPilot: identification focused on biological modifications, "thorough" search is engaged, iTRAQ 8-plex as sample type, MMTS as Cys alkylation reagent, trypsin as the digestion enzyme (see Note 21) and instrument type of 4800 MALDI TOF-TOF are selected. Protein detection threshold is set at an Unused ProtScore of 1.3, corresponding to $95.0 \%$ confidence interval (C.I.). The Paragon algorithm set instrument-appropriate mass error tolerance automatically. The iTRAQ reagent isotopic carryover bias correction is set as automatic.

2. In ProteinPilot software, in order to eliminate protein identification redundancy, raw peptide identification results are first processed by the Pro Group algorithm to create non-redundant protein groups from the peptides identified. A minimal set of proteins is produced for a given protein confidence interval threshold. In each group, one protein is designated as the winner protein for having the highest Unused ProtScore, meaning with the most number of peptides matched within the homologous group. 
3. To minimize the probability of false identification, consider only the winner proteins with an Unused ProtScore of at least 1.3 and having at least two distinct peptides with a minimum C.I. of $95 \%$ as identified.

4. To estimate the protein false discovery rate (FDR), all spectra are also searched against a decoy IPI Rat database containing all the same proteins with reversed sequences using the same search parameters as described above. The FDR is calculated as

$$
F D R=2 \times\left(N_{\text {decoy }}\right) /\left(N_{\text {decoy }}+N_{\text {forward }}\right)
$$

where $\mathrm{N}_{\text {decoy }}$ is number of proteins identified using the decoy database, $\mathrm{N}_{\text {forward }}$ is the number of proteins identified using the regular protein database (in this case Rat IPI protein database) $(13,24)$.

5. If the FDR is higher than the generally accepted $1.0 \%$, then repeat the search using higher Unused ProtScore until the FDR reach less than $1.0 \%$.

\subsubsection{Protein Quantification}

1. For relative protein quantification, the Pro Group algorithm calculates the relative protein expression ratios using only the iTRAQ ratios obtained from the peptide(s) that are distinct to each protein. The following peptides are excluded from quantification calculation; a) iTRAQ ion $\mathrm{S} / \mathrm{N}$ ratio $\leq 6$. b) C.I. $\%<1.0 \%$, c) peptides that are identified without an iTRAQ modification tag information, d) shared peptides - the spectrum matched to a peptide sequence that is claimed by more than one protein. 
2. The relative protein expression for each iTRAQ labeled sample is calculated as the weighted average for all the corresponding peptides.

3. Mean protein ratios are normalized for correcting the experimental bias introduced during labeling. Protein ratios for all iTRAQ labeled proteins are presented as the relative ratios compared to iTRAQ 113-labled proteins (see Note 22).

4. To calculate the average protein expression ratios between rapamycin-treated and the control samples, all eight quantification ratios for each protein is exported to Microsoft Excel and is calculated as follows: (average of the four rapamycin treated)/(average of the four control) values.

5. To identify differently expressed proteins, $\mathrm{p}$-values are calculated from the 2-tailed Student's T-test for each protein by comparing the four rapamycin treated OPCs ratios with the four control OPCs ratio values. Proteins exhibiting a greater than $20 \%$ changes $(\mathrm{p}<0.05)$ are considered significant (see Notes 23 and 24). These changes are beyond the $10 \%$ analytical coefficient of variance for ITRAQ analysis on our MS system (13).

\subsubsection{Alternate Protein Identification and Quantification Procedures}

Different database search algorithms identify only a fraction of the large number of spectra generally acquired during a shotgun proteomics experiment. They may also produce different identification and quantification results based on the identification cutoff criteria (25). It has been suggested that by using multiple search engines, a higher proportion of the proteome can be quantified $(26,27)$. Scaffold software is designed to analyze MS/MS-based protein identifications by comparing tandem mass spectrometry data that have been analyzed by the 
more than one search algorithms (e.g. Mascot, X! Tandem etc). To compare and validate the search results from multiple search engines; Scaffold uses PeptideProphet and ProteinProphet algorithms to assign statistical threshold for confidant protein identifications $(28,29)$.

Quantitative analysis of both peptide and protein changes can be further evaluated with the aid of the Scaffold Q+ 2.0 software module. The use of Scaffold analysis of Mascot and X! Tandem search results is described below.

1. Generate a peak list using TS2Mascot as a mascot generic file (MGF) from the tandem MS spectra using following parameters: mass range form 20-60 dalton below precursor, $\mathrm{S} / \mathrm{N}$ ratio of at least 10 .

2. Submit the peak list for automated protein sequence database search using a local Mascot server (version 2.3) against the rat IPI database (v3.55, Release date Feb 12, 2009, 39,874 sequence entries). Set following search parameters, iTRAQ 8-plex (K), iTRAQ 8-plex (N-teminal) and methylthio (C) as fixed modifications; iTRAQ 8-plex (Y) and Oxidation (M) as variable modifications; trypsin as the cleaving enzyme with maximum of one missed cleavage allowed; monoisotopic, peptide tolerance $50 \mathrm{ppm}$; MS/MS mass tolerance 0.3 Da. Reverse sequence database search was engaged. Once the search is completed, mascot generate a search result file as *.dat file for further analysis.

3. Open Scaffold, set up a new file, select quantitative technique as iTRAQ (8-plex), and insert the iTRAQ reagent purity correction parameters provided by AB SCIEX.

4. Load the *.dat file generated by Mascot and select the IPI protein database to be used for additional analysis by $\mathrm{X}$ ! Tandem, using the same search parameters as used for the Mascot search (see Note 25). 
5. After the search is completed, view the list of all the matched proteins in the sample pane of Scaffold. Set the minimum protein identification probability score to $95 \%$, minimum number of peptides to 2 and minimum peptide identification probability score to $95 \%$ (see Note 26).

6. Invoke the statistics pane in the Q+ module of Scaffold to evaluate the consistency of expression changes among different peptides belonging to the same protein (Fig. 2). If most of the light dots (each representing a peptide) fall near an optimal $45^{\circ}$ reporter ion correlation line drawn over the scatter plot of all the peptides identified from a control and rapamycin-treated sample, such observation represents an 1:1 ratio between the selected samples. Therefore, the protein is not differentially expressed. If the selected protein is up-regulated then the light spots representing the peptides within this protein on the scatter plot would have a slope greater than one. If the protein is down-regulated, the expected slope would be less than one (Fig. 2).

7. To obtain the relative quantification of proteins in each sample, invoke the Q+ module in Scaffold. Organize sample comparison by assigning the comparison categories: the iTRAQ labeled samples 113,114, 115 and 116 in the control category and iTRAQ labeled samples 117, 118, 119 and 121 in the rapamycin treated category.

6. Scaffold transforms quantitative ratios into $\log _{2}$ format and normalizes. Protein ratios for all iTRAQ-labeled proteins are presented as relative ratios compared to iTRAQ 113-labled proteins.

7. All eight quantification ratios for each protein can be exported to Microsoft Excel and converted back to anti-log fold changes. The relative protein expression between 
rapamycin treated and the control samples is calculated as follows: (average of the four rapamycin treated)/(average of the four control) values.

8. To identify differently expressed proteins, $\mathrm{p}$-values are calculated from the 2-tailed Student's T-test for each protein by comparing the four rapamycin treated OPCs ratios with the four control OPCs ratio values. 


\section{Notes}

1. Removal of residual media is very important. Otherwise, the components in cell culture media may interfere with subsequent digestion and iTRAQ labeling, resulting in external variability in the samples to be analyzed. We use PBS without any ammonium salt to completely wash the cells and to remove remaining media components. Traces of PBS should be removed by using a fine pipette tip, preferably with the gel loading tip without disturbing the cell pellets.

2. The lysis buffer described here has been successfully used to extract both soluble and membrane proteins. Other alternate buffers can also be used. One such buffer contains $20 \mathrm{mM}$ TEAB, $25 \mathrm{mM} \mathrm{Na} \mathrm{CO}_{3}$ and $0.1 \%(\mathrm{v} / \mathrm{v})$ of protease inhibitor cocktail, pH 8.5. Buffer based on urea can be also used. However, care must be taken to avoid buffers that contain primary amines (e.g. tris) which interfere with subsequent iTRAQ labeling.

3. It is very important to estimate the protein concentration of all the samples accurately. A protein concentration assay that is fully compatible with the lysis buffer should be employed. Here, we use the detergent-compatible BCA protein assay. For urea containing lysis buffer, the Bradford protein assay should be used.

4. As per iTRAQ manufacturer instruction, each protein sample should be between 5 and $100 \mu \mathrm{g}$ for each iTRAQ labeling reaction. To ensure, maximum labeling efficiency, sample volumes should not be more than $50 \mu$ leach. If the sample volume is larger than $50 \mu \mathrm{l}$, a speed vac or lyophilizer can be used to reduce the sample volume before iTRAQ labeling. To achieve effective reduction of protein disulfides, final concentration of TCEP should be maintained at $4 \mathrm{mM}$. If urea is used in lysis 
buffer, samples can be incubated at $37^{\circ} \mathrm{C}$ during the reduction step to avoid any carbamylation of amino acids (30). Final concentration of MMTS should be maintained at $8 \mathrm{mM}$ for effective alkylation of reduced cysteines.

5. For effective trypsin digestion, dilute the sample so that the final concentrations of the detergents and other chaotropic reagents do not inhibit trypsin activity.

6. Check the protein digestion efficiency before proceeding to iTRAQ labeling. Take 1 $\mu l$ from each of the digested samples. Cleanup the peptides with a SCX ziptip (Millipore, Billerica, Massachusetts). Mix the eluted peptides with the MALDI matrix solution in a 1:1 ratio and spot them onto a MALDI plate. Acquire the MS spectra and check if the peptide ion signals are comparable across all the eight samples. If the digestion is not complete, repeat the trypsin digestion step with additional enzyme.

7. Each iTRAQ tag may be provided in different volumes by vendor. It is normal if iTRAQ reagent volumes are not same in all the vials.

8. Make sure that the organic solvent concentration is at least $60 \%$ by adding HPLC grade isopropanol to the digested peptides before adding the iTRAQ solution for labeling. Lower organic concentration would result in rapid hydrolysis of iTRAQ reagents and poor labeling efficiency or no labeling.

9. For optimal labeling efficiency, the $\mathrm{pH}$ of the peptide/iTRAQ mixture solution must be between 7.5 and 8.5 . If the $\mathrm{pH}$ is less than 7.5 , the labeling efficiency would be significantly reduced.

10. Labeling with the 8-plex iTRAQ reagent requires a reaction time of $2 \mathrm{hrs}$ as compared to $1 \mathrm{hr}$ with the 4-plex version of the iTRAQ reagents. It is important to incubate the samples for at least $2 \mathrm{hrs}$ before combining in the next step. Failure to do 
so may result in incomplete labeling and artificial variations via cross-sample labeling.

11. Before combining the iTRAQ-labeled peptides, analyze an aliquot of each sample by MS/MS to verify the presence of each of the eight iTRAQ reporter ion peak in the spectra. First, remove the organic solvent by speed vac, and then clean the peptides using a SCX ZipTip. In MS/MS spectra, verify the presence of peaks at the $m / z$ of the appropriate iTRAQ reporter groups. If not, re-label the protein digests.

12. To remove the TEAB completely, re-suspend the dried sample in $250 \mu$ of HPLC grade water and dry again completely using speed vac. Repeat the process twice. Dried peptides can be stored at $-80^{\circ} \mathrm{C}$ till the next step.

13. After re-suspension of the peptides, make sure that concentration of the buffer salts is less than $10 \mathrm{mM}$ by diluting the sample mixture with $4.0 \mathrm{ml}$ of SCXLC mobile phase A.

14. To maximize the binding of the peptides to the column, samples should be loaded with relatively slow flow rate (e.g. $0.25 \mathrm{ml} / \mathrm{min}$ ). Alternatively the flow through can be re-loaded.

15. It is very important to wash the bound peptides with large volumes of mobile phase A to remove the detergents and residual iTRAQ reagents completely from the column prior to the start of elution gradient. Typically, wait until the UV absorbance reading reaches baseline before the initiation of the salt gradient.

16. At $\mathrm{pH}$ 3.0, basic residues in peptides (His, Arg, Lys) are positively charged, as are the $\mathrm{N}$-termini; acidic residues (Asp, Glu) are uncharged and the C-termini are mainly uncharged. Thus, most peptides with free N-termini will have net charges of at least 
+1 and should bind to PolySULFOETHYL A resin and can then be eluted with a salt gradient. Generally $2 / 3$ of all the peptides has +1 or +2 charge and is eluted with a gradient up to $0.25 \mathrm{M} \mathrm{KCl}$. The others typically have charges of +3 or +4 . It may be necessary to use a gradient to $0.6 \mathrm{M}$ salt to elute these peptides. In order to distribute tryptic peptides as uniformly as possible in the collected fractions we prefer to use a linear gradient with two segments; one segment goes to $0.25 \mathrm{M}$ salt which involves $75 \%$ of the gradient and a second segment from $0.25-0.6 \mathrm{M}$ salt with $\mathrm{pH} 4.5-6.0$ over the remaining $25 \%$ of the gradient.

17. For high salt concentration buffer fractions, $200 \mu 1$ of the loading solution may not be enough to dissolve the salts and peptides completely. Salts and peptides should be solubilized completely by adding more of the loading solution. Entire contents of the tube should be dissolved and loaded onto the spin column for desalting.

18. Volume of the peptide solutions to be loaded depends upon the concentrations of the peptides. In order to maintain peptide separation and resolution, saturating the reversed phase trap with excessive peptides should be avoided. The trapping column capacity for this protocol is $\sim 20$ pmoles. Absorbance at $214 \mathrm{~nm}$ during SCXLC fraction collections can be used as a guide to guage the concentration of peptides in each fraction. To further determine the complexity of the peptides in each fraction, mix an aliquot $(1.0 \mu \mathrm{l})$ of the desalted peptides with $1.0 \mu \mathrm{l}$ of the MALDI matrix. Acquire the MS spectra. SCXLC fractions whose MS spectra contain less than 100 peaks with $\mathrm{S} / \mathrm{N} \geq 5$ between $\mathrm{m} / z, 1000$ and 2000 and with a relatively low A214 absorbance during SCXLC (generally at the beginning and end of the SCXLC gradient) can be combined prior to subsequent RPLC separations. 
19. On each MALDI plate, eluted peptides from five different SCXLC fractions can be spotted, resulting in a total of 1650 MALDI spots. To avoid carry over between RPLC runs, a blank run should be performed between two RPLC runs to clean the chromatography system.

20. Turn on the high voltage for the MALDI source at least 30 min prior to update the default calibration. The warm-up period ensures the MS to deliver highest mass massaccuracy during the calibration step by reducing the variability in accelerating voltages and yielding more reproducible and accurate calibration results.

21. ProteinPilot software allows the search for tryptic, non-tryptic and semi-tryptic cleavages simultaneously. In conjunction with the iTRAQ technique, this feature has been successfully used to uncover regulated proteolytic events in spinal cords of experimental autoimmune encephalomyelitis animals by analyzing semi-tryptic and non-tryptic peptides independent from the tryptic peptides (11). This approach does not interfere with typical protein expression studies and may produce very useful information that is relevant to the underlying biology. This additional information may complement differential expression data commonly sought after by those performing shotgun proteomics studies. It can be obtained with relatively little additional effort and cost, thus improving proteomic research productivity.

22. In ProteinPilot, tag 113 is set as denominator for peptide ratio calculation by default. If it is required to set any other tag as the denominator, it can be selected under the quant function. Similarly, auto bias correction is set by default. However, in some cases, manual bias parameters can be set to compensate for extreme sample 
variability as a result of sample preparation artifacts and iTRAQ reagent heterogeneity.

23. Using the method described here, we acquired 54,485 MS/MS spectra. Total of 622 proteins were indentified with an estimated FDR of $0.1 \%$. Two hundred twenty one (221) proteins exhibited a greater than $20 \%$ change $(\mathrm{p}<0.05)$ in OPCs by rapamycin treatment.

24. It is very important to validate the iTRAQ quantification results using alternate methods including Western blotting, immunohistochemistry or other biological methods.

25. Scaffold needs to access the same protein sequence database that is used by Mascot search. Upload the same database (Rat IPI protein database in this case) into Scaffold beforehand.

26. Minimum protein identification probability set the threshold requirement for Scaffold to calculate the probability of correct protein identification. Minimum number of peptides set the number of unique peptides that must be found for a given protein in order to consider the protein to be identified. Minimum peptide identification probability set how certain (C. I. \%) a peptide identification must be before it can be counted toward the minimum number of peptides. 


\section{References}

1. Noorbakhsh F, Overall CM, Power C (2009) Deciphering complex mechanisms in neurodegenerative diseases: the advent of systems biology. Trends Neurosci 32: 88-100

2. Gygi SP, Rochon Y, Franza BR et al (1999) Correlation between protein and mRNA abundance in yeast. Mol Cell Biol 19: 1720-1730

3. Bayes A, Grant SG (2009) Neuroproteomics: understanding the molecular organization and complexity of the brain. Nat Rev Neurosci 10: 635-646

4. Colucci-D'Amato L, Farina A, Vissers JP et al (2010) Quantitative Neuroproteomics: Classical and Novel Tools for Studying Neural Differentiation and Function. Stem Cell Rev doi:10.1007/s12015-12010-19136-12013

5. Gygi SP, Rist B, Gerber SA et al (1999) Quantitative analysis of complex protein mixtures using isotope-coded affinity tags. Nat Biotechnol 17: 994-999

6. Fu C, Hu J, Liu T et al (2008) Quantitative analysis of redox-sensitive proteome with DIGE and ICAT. J Proteome Res 7: 3789-3802

7. Fu C, Wu C, Liu T et al (2009) Elucidation of thioredoxin target protein networks in mouse. Mol Cell Proteomics 8: 1674-1687

8. Ong SE, Blagoev B, Kratchmarova I et al (2002) Stable isotope labeling by amino acids in cell culture, SILAC, as a simple and accurate approach to expression proteomics. Mol Cell Proteomics 1: 376-386

9. Ross PL, Huang YN, Marchese JN et al (2004) Multiplexed protein quantitation in Saccharomyces cerevisiae using amine-reactive isobaric tagging reagents. Mol Cell Proteomics 3: 1154-1169 
10. Liu T, Donahue KC, Hu J et al (2007) Identification of differentially expressed proteins in experimental autoimmune encephalomyelitis (EAE) by proteomic analysis of the spinal cord. J Proteome Res 6: 2565-2575

11. Jain MR, Bian S, Liu T et al (2009) Altered proteolytic events in experimental autoimmune encephalomyelitis discovered by iTRAQ shotgun proteomics analysis of spinal cord. Proteome Sci 7: 25

12. Grant JE, Hu J, Liu T et al (2007) Post-translational modifications in the rat lumbar spinal cord in experimental autoimmune encephalomyelitis. J Proteome Res 6: 27862791

13. Hu J, Qian J, Borisov O et al (2006) Optimized proteomic analysis of a mouse model of cerebellar dysfunction using amine-specific isobaric tags. Proteomics 6: 4321-4334

14. Liu T, Hu J, Li H (2009) iTRAQ-based shotgun neuroproteomics. Methods Mol Biol 566: $201-216$

15. Jain MR, Liu T, Hu J et al (2008) Quantitative Proteomic Analysis of Formalin Fixed Paraffin Embedded Oral HPV Lesions from HIV Patients. Open Proteomics J 1: 40-45

16. Hunt DF, Yates JR, 3rd, Shabanowitz J et al (1986) Protein sequencing by tandem mass spectrometry. Proc Natl Acad Sci U S A 83: 6233-6237

17. Steen H, Mann M (2004) The ABC's (and XYZ's) of peptide sequencing. Nat Rev Mol Cell Biol 5: 699-711

18. Sarbassov DD, Ali SM, Sabatini DM (2005) Growing roles for the mTOR pathway. Curr Opin Cell Biol 17: 596-603

19. Kim DH, Sarbassov DD, Ali SM et al (2002) mTOR interacts with raptor to form a nutrient-sensitive complex that signals to the cell growth machinery. Cell 110: 163-175 
20. Sarbassov DD, Ali SM, Kim DH et al (2004) Rictor, a novel binding partner of mTOR, defines a rapamycin-insensitive and raptor-independent pathway that regulates the cytoskeleton. Curr Biol 14: 1296-1302

21. Tyler WA, Gangoli N, Gokina P et al (2009) Activation of the mammalian target of rapamycin (mTOR) is essential for oligodendrocyte differentiation. J Neurosci 29: 63676378

22. Bibollet-Bahena O, Almazan G (2009) IGF-1-stimulated protein synthesis in oligodendrocyte progenitors requires PI3K/mTOR/Akt and MEK/ERK pathways. J Neurochem 109: 1440-1451

23. Shilov IV, Seymour SL, Patel AA et al (2007) The Paragon Algorithm, a next generation search engine that uses sequence temperature values and feature probabilities to identify peptides from tandem mass spectra. Mol Cell Proteomics 6: 1638-1655

24. Peng J, Elias JE, Thoreen CC et al (2003) Evaluation of multidimensional chromatography coupled with tandem mass spectrometry (LC/LC-MS/MS) for largescale protein analysis: the yeast proteome. J Proteome Res 2: 43-50

25. Kapp EA, Schutz F, Connolly LM et al (2005) An evaluation, comparison, and accurate benchmarking of several publicly available MS/MS search algorithms: sensitivity and specificity analysis. Proteomics 5: 3475-3490

26. Resing KA, Meyer-Arendt K, Mendoza AM et al (2004) Improving reproducibility and sensitivity in identifying human proteins by shotgun proteomics. Anal Chem 76: 35563568

27. Elias JE, Haas W, Faherty BK et al (2005) Comparative evaluation of mass spectrometry platforms used in large-scale proteomics investigations. Nat Methods 2: 667-675 
28. Keller A, Nesvizhskii AI, Kolker E et al (2002) Empirical statistical model to estimate the accuracy of peptide identifications made by MS/MS and database search. Anal Chem 74: 5383-5392

29. Nesvizhskii AI, Keller A, Kolker E et al (2003) A statistical model for identifying proteins by tandem mass spectrometry. Anal Chem 75: 4646-4658

30. Stark GR, Stein WH, Moore S (1960) Reactions of the Cyanate Present in Aqueous Urea with Amino Acids and Proteins. J Biochem Mol Biol 235: 3177-3181 


\section{Figure Captions}

Fig. 1 iTRAQ-based expression proteomics work flow for identification of proteins regulated by the mTOR pathway during oligodendrocyte differentiation.

Proteins extracted from four control and four rapamycin-treated OPCs groups are sequentially reduced, alkylated and digested by trypsin. The resulting peptides are individually labeled with the iTRAQ reagents $113,114,115,116,117,118,119$ and 121 as indicated. The labeled peptides are combined and fractionated using SCXLC and RPLC (2D LC/MS/MS) and analyzed on a tandem mass spectrometer. Database search and bioinformatics procedures are used for protein (A) quantification and (B) identification.

Fig. 2 Down-regulation of isocitrate dehydrogenase is ascertained from iTRAQ analysis by Scaffold.

(A) Representative Scaffold graphical plot of normalized reporter ion intensity values of iTRAQ reporter ion 113 (see Fig.1, Control 1) versus those of iTRAQ reporter ion 117 (see Fig. 1, Rapamycin treatment 1) for all the identified peptides is shown (dark dots). The iTRAQ plot dots fall near the center straight line represent the peptides that are not differentially regulated by ramamycin treatment. Each light dot represents the iTRAQ signals derived from a peptide matched to isocitrate dehydrogenase. iTRAQ ion plots of various intensities derived from the tryptic peptides obtained from this protein revealed similar down-regulated iTRAQ expression trend of $117 / 113$ ratios of $\sim 0.7$. (B) A bar graph of the normalized intensities of the iTRAQ reporter ions for a representative peptide ion (circled and pointed with arrow in A) of isocitrate dehydrogenase indicates consistent down-regulation of this peptide ( $\mathrm{R} / \mathrm{C}$ of $\sim 0.7$ ) across all the 
replica of the experiment (C1-C4 represents the control samples and $\mathrm{R} 1-\mathrm{R} 4$ represents rapamycin treated samples (see Fig. 1). (C) Continuous series of the $\mathrm{b}$ and $\mathrm{y}$ ions allows the identification of the peptide as DIFQEIYDK (circled in A). (D) A bar graph of the means and standard deviations of the protein level iTRAQ ratios in relation control signal obtained from all the peptides derived from isocitrate dehydrogenase. Quantification variability among the peptides is shown by the error bars. Notably the means for each independent experiment are very consistent among the replicates. 
Control OPCs

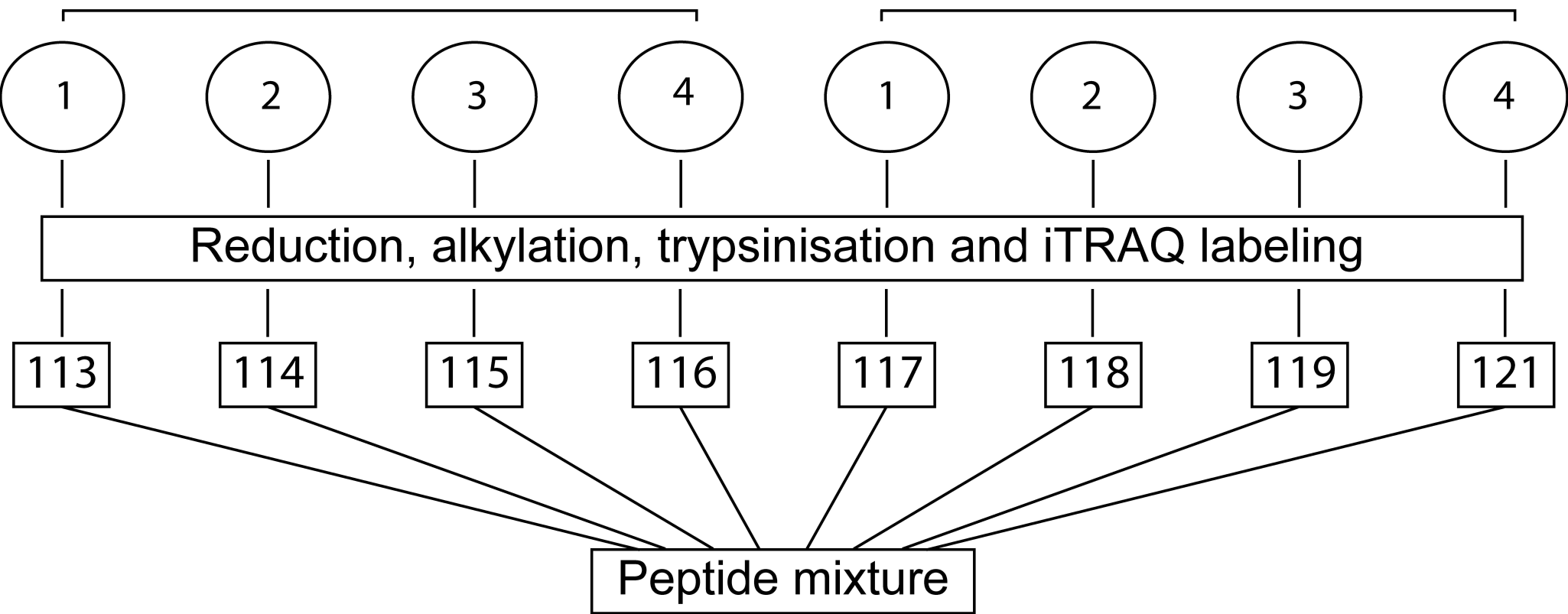

\section{Protein quantification and identification}

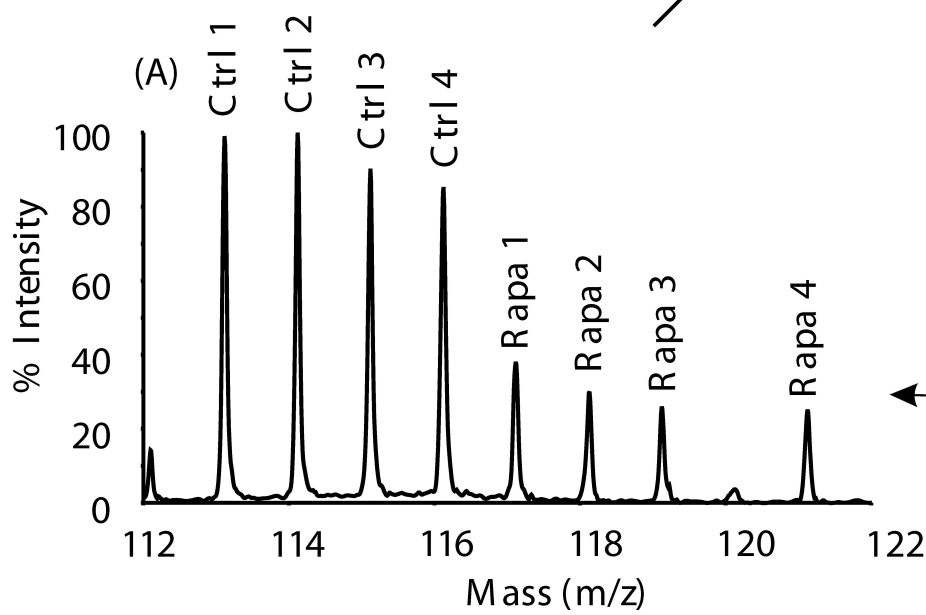

(B)

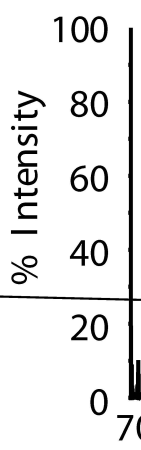

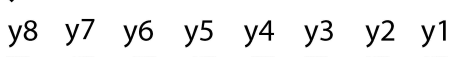
$F-S-W-G-A-E-G-Q-K$ b1 $\begin{array}{lllllll}\mathrm{b} 2 & \mathrm{~b} 3 & \mathrm{~b} 4 & \mathrm{~b} 5 & \mathrm{~b} 6 & \mathrm{~b} 8\end{array}$

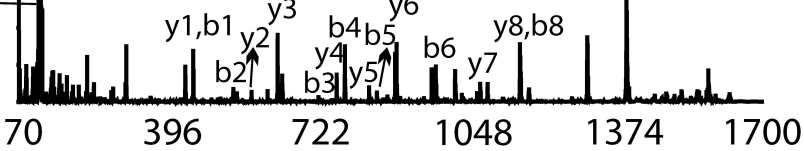
Mass (m/z) 


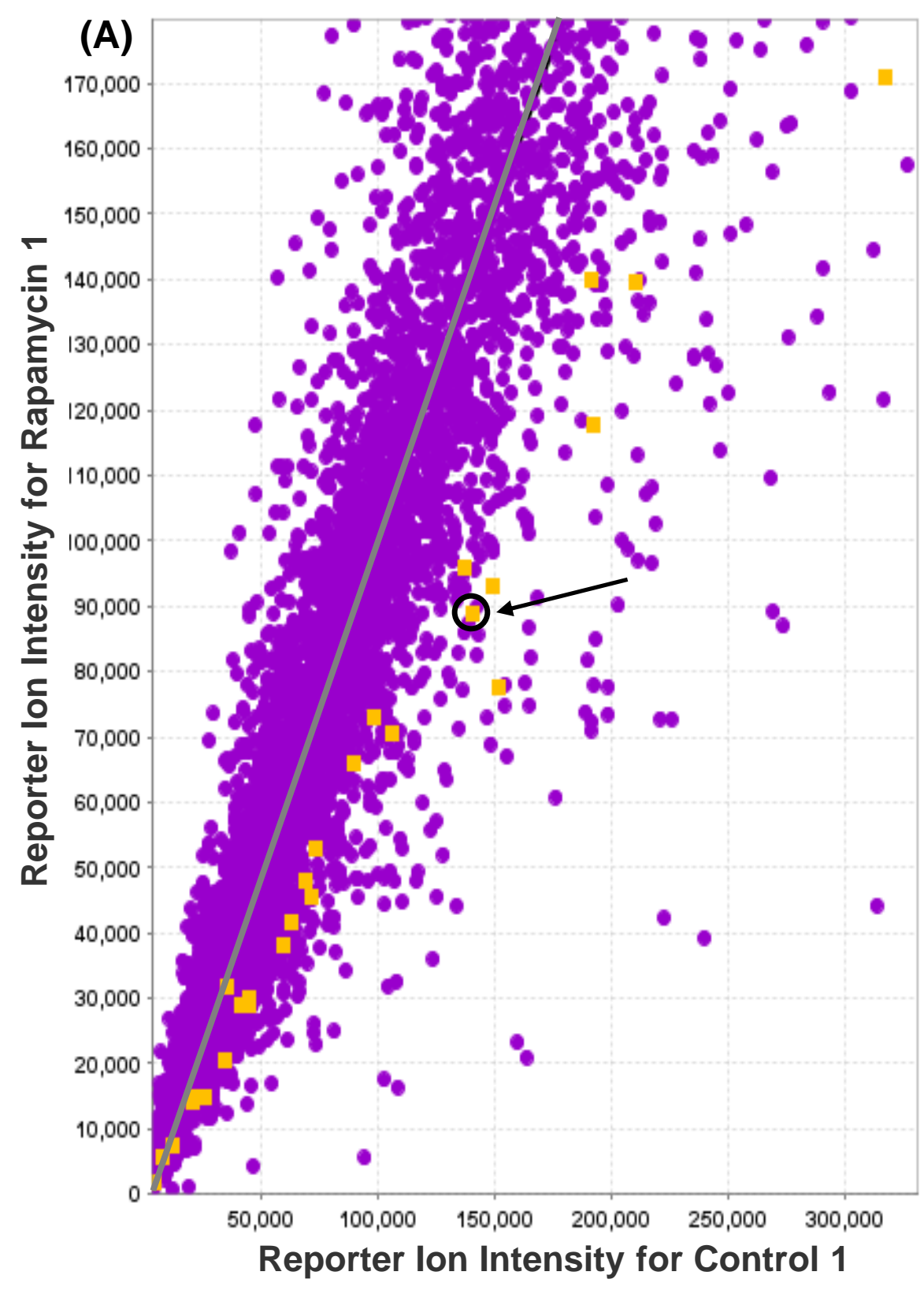

(B)

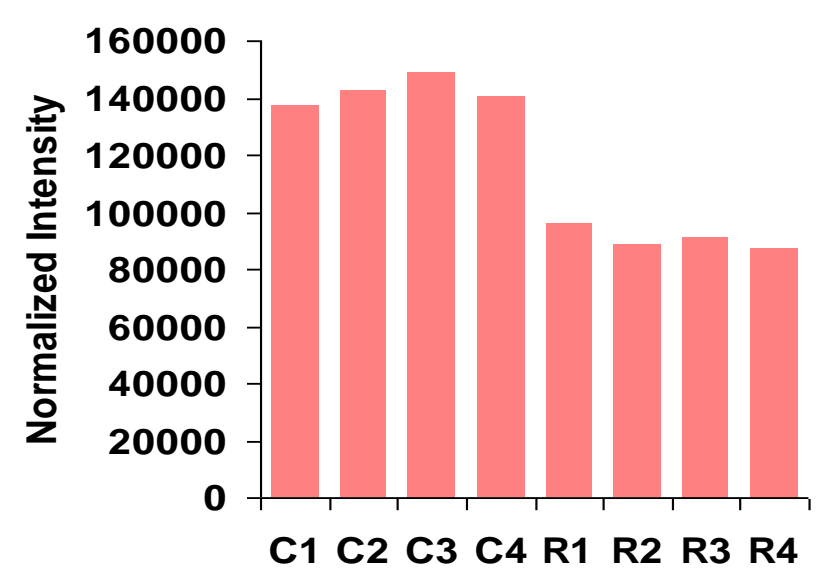

(C) DIFQEIYDK
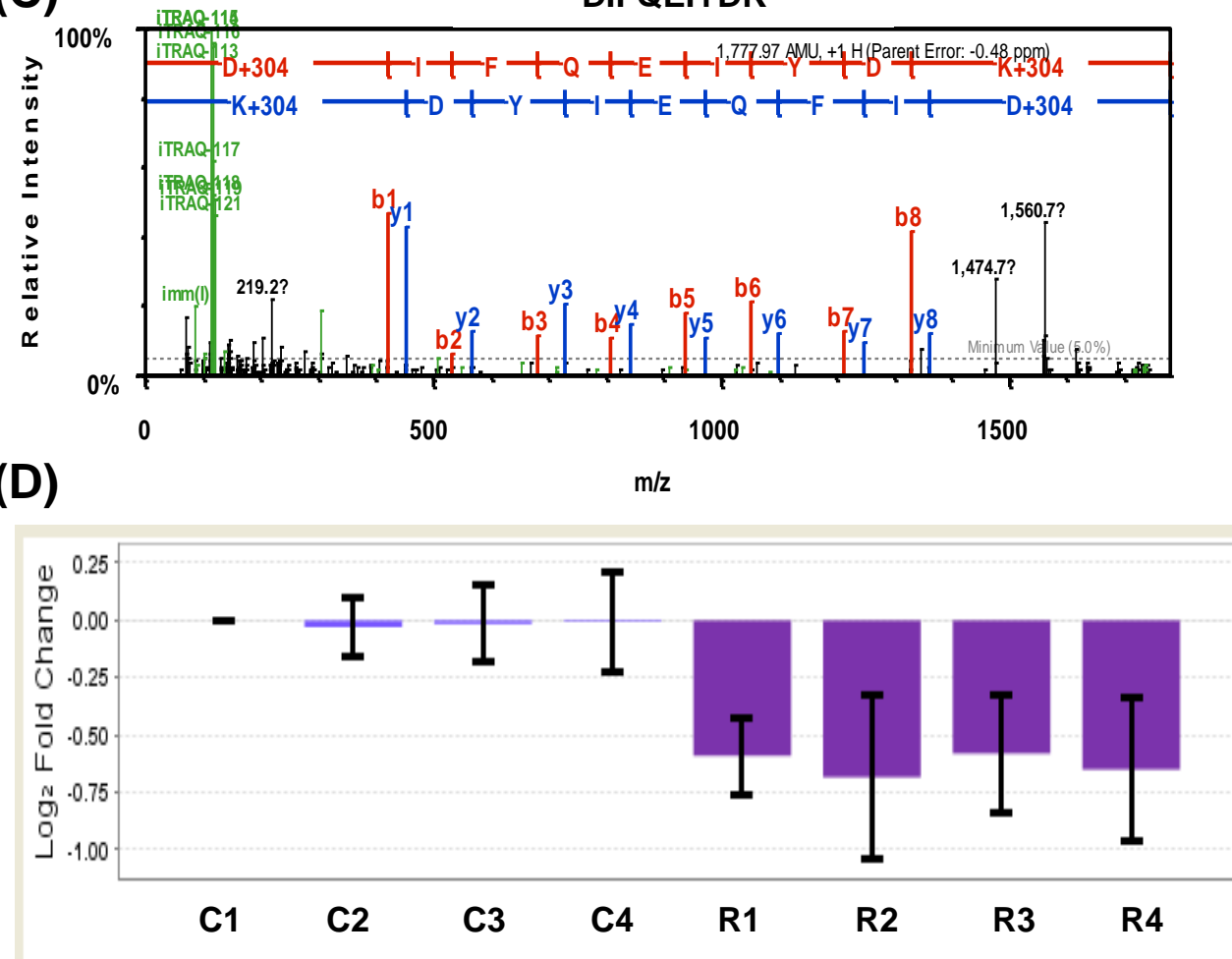

IPI00194045 - Others - Optimal $45^{\circ}$ Sample Correlation 